\title{
Dictynna
}

Dictynna

Revue de poétique latine

$4 \mid 2007$

Varia

\section{Ève et le serpent, une réécriture chrétienne de la rencontre entre Médée et Jason}

Approche intertextuelle du récit de la tentation dans l'Histoire spirituelle d'Avit de Vienne (2, 204-231)

Nicole Hecquet-Noti

\section{OpenEdition}

Journals

Édition électronique

URL : http://journals.openedition.org/dictynna/148

DOI : 10.4000/dictynna. 148

ISSN : 1765-3142

Référence électronique

Nicole Hecquet-Noti, « Ève et le serpent, une réécriture chrétienne de la rencontre entre Médée et Jason », Dictynna [En ligne], 4 | 2007, mis en ligne le 29 novembre 2010, consulté le 10 décembre 2020. URL : http://journals.openedition.org/dictynna/148; DOI : https://doi.org/10.4000/dictynna. 148

Ce document a été généré automatiquement le 10 décembre 2020.

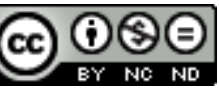

Les contenus des la revue Dictynna sont mis à disposition selon les termes de la Licence Creative Commons Attribution - Pas d'Utilisation Commerciale - Pas de Modification 4.0 International. 


\title{
Ève et le serpent, une réécriture chrétienne de la rencontre entre Médée et Jason
}

\author{
Approche intertextuelle du récit de la tentation dans l'Histoire \\ spirituelle d'Avit de Vienne (2, 204-231)
}

\section{Nicole Hecquet-Noti}

1 Au livre 2 de son Histoire spirituelle, Avit présente une très longue scène dramatique où est narrée avec force détails et innovations par rapport au texte, relativement succinct, de la Genèse la tentation d'Ève par le serpent ${ }^{1}$. Située au cœur de l'ensemble formé par les trois premiers chants consacrés à Adam en tant que préfiguration du Christ, nouissimus $\mathrm{Adam}^{2}$, ce récit a été magistralement analysé par P. A. Deproost dans un récent article ${ }^{3}$. Je voudrais proposer ici une analyse complémentaire à cet article en abordant un point de vue différent de celui de Deproost qui met en évidence avant tout les procédés littéraires et rhétoriques qu'utilise le poète pour composer une scène dramatique. Je m'intéresserai, pour ma part, au noyau central de ce passage pour montrer comment, après la longue approche rhétorique du serpent et le dialogue entre Ève et son séducteur, Avit décrit, avec de minutieux détails, la scène même de la tentation, c'est-à-dire le moment où le serpent abandonne sa "rhétorique de la persuasion" pour passer à une "gestuelle de la persuasion" afin de vaincre définitivement sa victime (v. 204-231).

De fait, si, dans cette description, Avit a principalement pour source le texte biblique et l'Énéide, il me semble que le modèle de référence doit plutôt être recherché du côté de la poésie érotique, en particulier dans la poésie élégiaque, avec laquelle le poète entretient un dialogue constant mais discret que je chercherai à expliquer en le replaçant aussi bien dans le contexte littéraire du poète que dans l'environnement théologique de l'évêque. 


\section{Le récit de la tentation chez les poètes chrétiens}

3 À l'exception de l'Histoire spirituelle, l'épisode de la tentation, moment théologiquement crucial, puisqu'il introduit le péché originel dans la race humaine, est brièvement évoqué dans les autres épopées bibliques s'inspirant de la Genèse (PS. CYPRIEN, Genesis 72-88; MARIUS VICTOR, Alethia, 1, 393-423 et DRAConTIUs, De laudibus Dei, 1, 459-4844) suivant un schéma identique : dans le jardin d'Eden, dont ils ont rappelé auparavant la luxuriance et les délices, apparaît le serpent qui va séduire Ève par des paroles très persuasives $^{5}$. Curieusement toutefois, aucun poète ne décrit en détail le geste d'Ève se saisissant du fruit.

4 Dans son austère épopée ${ }^{6}$, le Pseudo Cyprien se contente de suivre le texte biblique : après les paroles du serpent, Ève qui, tout d'abord, refuse de toucher au fruit défendu, finit tout de même par le mordre : en quatre vers, le poète évoque les hésitations de la femme et son geste, sans donner aucun détail précis :

illa negat uetitosque timet contingere ramos

sed tamen infirmo uincuntur pectora sensu.

ilicet ut niueo iam mitia dente momordit

adfulsit nulla maculatum nube serenum. ${ }^{7}$

Il en va de même dans les épopées de Marius Victor et de Dracontius dont le style est pourtant plus ornés. Dans l'Alethia, après les paroles quelque peu développées du serpent $(1,398-405 a)$, et un jugement moral porté par le poète lui-même sur cet épisode (1, 405b-411a), Marius Victor ne décrit nullement le geste d'Ève, mais préfère insister sur la participation d'Adam et sur les conséquences du péché pour les deux êtres humains (1,411-423), seuls deux vers et demi évoquant précisément la tentation d'Ève $(1,411 b-414 a)$ :

...nam credula postquam

rupit sacrilegis praescriptum morsibus Eua

experti iam docta mali, solacia culpae

quaerit ${ }^{9}$.

Dracontius, dans le De laudibus Dei $(1,459-484)$ ne donne même pas la parole au serpent, contrairement à ces prédécesseurs, mais relate l'entier de l'épisode dans un récit qui, comme celui de Marius Victor, évoque très rapidement, en un seul vers, le geste d'Ève :

...coniugis aures

aggreditur sub uoce pia, sermone maligno

insidiosus adit heu mollia corda puellae:

ingerit ore cibos crudeli funere plenos. ${ }^{10}$

7 Avit quant à lui consacre 28 vers à une minutieuse peinture de cette scène dramatique, amplifiant ainsi considérablement le récit biblique de Genèse 3,6:

Talia fallaci spondentem dona susurro /credula submisso miratur femina uultu. /Et iam iamque magis cunctari ac flectere sensum /incipit et dubiam leto plus addere mentem. /Ille ut uicino uictam discrimine sensit, / atque iterum nomen memorans arcemque deorum / unum de cunctis letali ex arbore malum / detrahit et suaui pulchrum perfundit odore. / Conciliat speciem nutantique insuper offert, / nec spernit miserum mulier male credula munus, / sed capiens manibus pomum letale retractat. / Naribus interdum labiisque patentibus ultro / iungit et ignorans ludit de morte futura. / 0 quotiens ori admotum compuncta retraxit / audacisque mali titubans sub pondere dextra / cessit et effectum sceleris tremefacta refugit!/ Dis tamen esse cupit similis serpitque uenenum / ambitione nocens. Rapiunt contraria mentem / hinc amor, inde metus. Pulsat iactantia legem / interdumque etiam lex subuenit. Aestuat anceps / 
diuidui cordis dura inter proelia fluctus. / Nec tamen incentor desistit fallere serpens / ostentatque cibum dubiae queriturque morari / et iuuat in lapsum pendentis prona ruinae. / Vt tandem uictae grauior sententia sedit / aeternam temptare famem per criminis escam, / serpentem satiare cibo, quem sumeret ipsa, / adnuit insidiis pomumque uorata momordit ${ }^{11}$.

\section{Comment et pourquoi Avit a-t-il ainsi amplifié le récit biblique?}

P. A. Deproost a déjà apporté un élément de réponse en montrant la volonté du poète de mettre en scène le drame intérieur ${ }^{12}$ de la femme en utilisant les ressources de la rhétorique dont l'emprise est considérable dans la poésie tardive. Toutefois, ce qui m'intéresse ici ce n'est pas le long dialogue entre Ève et le serpent, mais la description du moment précis où Ève enfin séduite va commettre le geste fatal. Là encore, le poète innove considérablement aussi bien par rapport au récit biblique que par rapport aux épopées antérieures. Par ce petit tableau, il introduit dans son épopée les caractéristiques de l'expression de la poésie érotique en construisant une véritable scène de séduction digne de la poésie élégiaque comme le montre l'analyse des différents éléments de ce passage.

\section{Le cadre}

9 Conformément à ce que dit la Genèse, c'est dans le paradis que se déroule la tentation. Toutefois, Avit ne décrit pas le jardin dans le chant 2, car il a consacré toute la fin du chant précédent à une très longue et minutieuse description de cet hortus deliciarum (1, 191-298) qui s'inscrit dans la tradition littéraire du locus amoenus ${ }^{13}$. Il se contente de rappeler cette description dès le début de la scène de la tentation en montrant le bonheur insouciant des nouveaux hommes occupés à cueillir les pommes rouges d'un vert rameau $(2,138 b-139)$ :

...nam forte rubentia laeti

carpebant iuuenes uiridi de palmite mala.

Dans ce détail non scripturaire, l'expression rubentia mala rappelle les vers de la Copa qui évoquent de manière très sensuelle les appâts de séductrice de la "cabaretière " invitant le passant à s'arrêter dans son auberge, véritable hortus deliciarum, où parmi tous les éléments caractéristiques du locus amoenus, se trouvent les mêmes fruits de l'automne qu'offre Tityre à Mélibée à la fin de la première bucolique virgilienne :

Sunt et caseoli, quos iuncea fiscina siccat.

Sunt autumnali cerea pruna die,

castaneaeque nuces et suaue rubentia mala ${ }^{14}$.

11 Par cette discrète allusion, Avit situe donc la tentation dans un cadre lyrique propre à l'expression des sentiments amoureux et il justifie déjà son choix de la pomme en tant que fruit défendu, point sur lequel je vais revenir. C'est aussi dans un cadre identique où se mêlent fleurs et fruits spontanément produits comme dans le jardin d'Ėden qu'Hylas cueille en toute insouciance des fruits avant d'être enlevé par les Dryades ${ }^{15}$ dans une élégie où Properce avertit Gallus de veiller sur ses amours qui peuvent être convoités par autrui. 

chant 2 , elle est toutefois bien présente et sous-jacente à l'ensemble de la scène grâce à un réseau d'allusions à la description du chant 1 : outre les rubentia mala qui font écho à l'abondance paradisiaque et, en particulier, à la présence constante de fruits d'automn $\mathrm{e}^{16}$, le suauis odor de la pomme fatale renvoie aux suaues odores qui embaument le paradis ${ }^{17}$. Or, les parfums sont un des éléments essentiels de la sensualité dans la poésie érotique, qu'ils soient utilisés comme moyen de séduction en enveloppant une personne ou qu'ils agrémentent le décor ${ }^{18}$. En particulier, on retrouve la même expression suaues odores dans la même position métrique dans un vers où Virgile évoque le cadre bucolique dans lequel Corydon exprime son amour désespéré pour le bel Alexis :

ego cana legam tenera lanugine mala

castaneasque nuces, mea quas Amaryllis amabat;

addam cerea pruna (...)

et uos, o lauri, carpam et te, proxime myrte,

sic positae quoniam suauis miscetis odores ${ }^{19}$.

Or, dans la description de Virgile, on retrouve aussi, comme dans la Copa ou dans Ecl. 1, l'énumération de fruits (parmi lesquels il y a des mala) cueillis par l'être amoureux. Il semble bien, d'après l'expression tenera lanugine qui les qualifie, qu'il s'agit de coings et non de pommes. Toutefois même si la nature réelle du fruit est sujette à discussion, on a dans la pièce de Virgile tous les ingrédients qu'Avit a repris pour évoquer le cadre de son récit de la tentation.

\section{Les personnages}

Outre le cadre de la tentation qui évoque par des touches allusives précises et choisies le décor de l'expression de la poésie érotique, les protagonistes eux aussi ne sont pas sans liens directs avec les personnages de la poésie érotique.

$\mathrm{Au}$ début de la scène (v. 136-139), nous sommes en présence de trois personnes: le diable, qui a pris l'apparence du serpent, Adam et Ève, le premier couple, occupés à cueillir des pommes. Or, on apprend tout de suite que le serpent va bouleverser ce couple, puisqu'il désire subuertere corda, en s'adressant non pas à l'homme dont l'esprit est ferme, mais à la femme, plus faible ${ }^{20}$. Ainsi sont déjà esquissées les interactions qui existent entre ces trois personnages, qui rappellent le «triangle » mari, femme, amant de la poésie érotique. Nous sommes face à la situation type de l'élégie où l'amant / serpent dirige l'action en tentant de séduire la femme, loin de son mari.

Le mari, Adam, est totalement absent de la scène qui va se dérouler. Si, au début, il cueille des pommes avec sa femme, il tombe dans l'oubli immédiatement après que le serpent a préféré séduire cette dernière. Comme dans la Genèse, le poète n'explique pas son absence, il ne le fait pas sortir de scène, mais, après la tentation d'Ève, il le fait revenir vers sa femme, suggérant ainsi qu'il s'en est éloigné auparavant ${ }^{21}$. Contrairement à la poésie élégiaque dans laquelle, même s'il est absent, le mari est un protagoniste souvent présent en filigrane, voire un obstacle pour l'amant jaloux qui voit en lui un rival ${ }^{22}$, ici, il n'interfère d'aucune façon. Deproost voit dans cet effacement d'Adam une façon pour le poète de « dégager Adam de la pleine conscience d'une faute qu'il n'avait pas commise le premier, qui s'était d'abord produite en son absence, et dont il ne mesurait pas toutes les implications $»^{23}$. 
17 En effet, si on suit l'analyse d'Augustin, Adam, créé à l'image de Dieu, ne peut être tenté charnellement, il a donc besoin d'un intermédiaire plus faible ${ }^{24}$. Ève, la victime du serpent, est présentée comme faible et "pouvant être séduite " par le néologisme seductilis $(2,166)$ qu'Avit a repris à Augustin ${ }^{25}$. De fait, cet adjectif, formé sur le verbe seducere a une connotation très forte en indiquant une coupure qui isole une personne de sa communauté, comme l'attestent les rares emplois du participe seductus appliqué à une personne ${ }^{26}$. La faiblesse de la femme, facilement encline à céder aux avances d'un homme, est la conséquence d'une condamnable crédulité, ce que ne manque pas de rappeler Hélène lorsqu'elle s'adresse à Pâris (ov., Epist. 17, 41) :

credulitas damno solet esse puellis..

18 Cette néfaste credulitas est le propre de toute femme amoureuse comme le dit encore Ovide dans un passage à la tonalité élégiaque lorsqu'il qualifie l'attitude de Procis ajoutant foi aux paroles ambiguës de Céphale, rapportées par la brise : credula res amor est (Met. 7, 826) ${ }^{27}$. Ainsi, Médée, avec lucidité, comprend bien que c'est cette confiance excessive en l'amour trompeur de Jason qui l'a perdue (ov. Epist. 12, 120) :

$\mathrm{Tu}$ fraudis poenas, credulitatis ego ${ }^{28}$,

comme elle perdra Ève dont Avit, sur le modèle de Marius Victor ${ }^{29}$, souligne, dès le début du récit de la tentation, la crédulité ${ }^{30}$. Dans le chant 3 , Adam se défendra face à Dieu, en invoquant justement la confiance qu'il avait en sa femme comme Dieu luimême le lui avait demandé lors de la création ${ }^{31}$.

19 Ainsi donc, il semble bien que ce soit à cause de ses sens que la femme est le plus vulnérable : or, toute la poésie érotique joue sur cela et Avit fait de même dans notre passage. En effet, le serpent commence certes par attaquer Ève par un long discours persuasif (v. 185-203), mais, les paroles ne suffisent pas et il doit encore flectere sensum (v. 206) de sa victime. Dans ce contexte, il ne faut pas prendre sensus comme un synonyme de mens, mais en opposition à cette dernière ${ }^{32}$ pour distinguer les sens de l'esprit rationnel : la tentation deviendra effective au moment où les sens d'Ève auront été pervertis, ce qui ne sera possible que grâce à la pomme. Fléchir les sens de la femme pour s'assurer de son amour est un lieu commun de la poésie érotique : on retrouve en particulier l'expression, en fin de vers comme dans notre passage, dans les Argonautiques de Valerius Flaccus $(7,172)$ : Médée lutte contre l'amour qu'elle éprouve pour Jason au grand dam de Junon qui demande à Vénus de la faire succomber et quitter son père pour suivre Jason. Vénus s'exécute et répond à Junon :

nec tibi cum primos adgressa es flectere sensus

uirginis ignotaque animum contingere cura

defuimus ${ }^{33}$.

20 Comme dans notre passage, il s'agit de faire basculer une femme hésitante, partagée entre un amour filial, légitime, et une passion sensuelle et dangereuse qui l'amènera à sa perte ${ }^{34}$. C'est précisément ce combat entre obéissance et passion qui est amplement développé par Avit dans les vers 206-225, sur le modèle des hésitations des femmes amoureuses dans la poésie classique :

\footnotetext{
... Rapiunt contraria mentem

hinc amor, inde metus. Pulsat iactantia legem

interdumque etiam lex subuenit. Aestuat anceps

diuidui cordis dura inter proelia fluctus. ${ }^{35}$
}

$21 \mathrm{Si}$, comme le signalent plusieurs emprunts au chant 4 de l'Énéide ${ }^{36}$, le modèle premier en est Didon hésitant face son amour pour Ėnée, le personnage d’Ève est aussi la 
synthèse de nombre de femmes que leur passion amoureuse va exclure de la communauté : les hésitations d'Ève avant de transgresser l'interdit émis par Dieu ne sont pas sans lien avec la confusion de Byblis en proie à un amour interdit pour son frère. On trouve en effet l'expression dubiam mentem au même endroit dans le v. 207 d'Avit :

incipit et dubiam leto plus addere mentem

et dans le vers concluant le discours hésitant de Byblis effrayée devant les conséquences de son amour interdit (ov. Met. 9, 517):

hoc placet, haec dubiam uicit sententia mentem ${ }^{37}$

Le même verbe aestuare est utilisé par les deux poètes pour exprimer la frénésie de la passion $^{38}$. En outre, les atermoiements d'Ève au moment de prendre la pomme sont introduits par la même expression o quotiens que l'on rencontre pour exprimer l'hésitation dans la poésie élégiaque ${ }^{39}$, et surtout, dans les Métamorphoses au début de la partie décrivant, dans un style très élégiaque, Atalante dans son duel face à Hippomèn $e^{40}$. Les deux histoires présentent plusieurs points de convergence. En effet, comme Ève face au serpent, Atalante hésite face au défi lancé par Hippomène, lequel ne la vaincra que par la ruse en l'obligeant à ramasser la pomme donnée par Vénus et à retarder ainsi sa course : c'est le poids de la pomme qui va sceller la défaite d'Atalante. On peut voir ici un jeu allusif, tout à fait dans l'esprit maniéré de la poésie tardive, entre le vers d'Ovide (Met. 10, 677) :

tollere et adieci sublatopondera $m \bar{a} \overline{l o}$

et le vers d'Avit :

audacisque măli titubans sub pondere dextra

En effet, nous avons là un jeu de mots entre mālum, la pomme dans le vers d'Ovide, et mălum, le mal, dans le vers d'Avit qui, auparavant a introduit lui-même ce jeu de mots sur lequel je vais revenir ${ }^{41}$.

Outre ces rapprochements contextuels entre Éve et les héroïnes amoureuses de la poésie classique, il reste à signaler que l'expression quasi proverbiale du début du vers 222 hinc amor, inde metus renvoie elle aussi à ces femmes évoquées. Si les mêmes mots ne sont pas utilisés dans la poésie classique, en revanche on trouve des expressions synonymes à propos des femmes amoureuses. Ovide utilise le groupe hinc amor, hinc timor (qui permet une paronomase) en tête de vers dans Epist. 12, 63 $3^{42}$. L'opposition entre amor et timor est aussi au centre des interrogations exprimées par Médée dans Met. 7, 11-16:

... «Frusta, Medea repugnas;

Nescio qui deus obstat; ; ait « mirumque nisi hoc est

aut aliquid certe simile huic quod amare uocatur.

Nam cur iussa patris nimium mihi dura uidentur?

Sunt quoque dura nimis. Cur quem modo denique uidi

ne pereat timeo ? quae tanti causa timoris ? ${ }^{43}$

Si Médée éprouve des sentiments confus d'amour et de crainte pour Jason, elle souffre aussi de la dureté des ordres de son père qui a imposé les épreuves à Jason. Or, c'est par une expression identique que le serpent-tentateur qualifie, de sa voix caressante, l'interdiction émise par Dieu (2,159-160) :

Scire uelim : quis dura iubet, quis talia dona inuidet...

Dans sa lettre, Médée adresse des reproches à Jason qu'elle décrit comme un être fourbe et rusé en usant des mots qui rappellent les qualificatifs utilisés par Avit pour le 
serpent ${ }^{44}$, esquissant ainsi un renversement des situations des personnages entre les deux histoires: en effet, comme le relève Valerius Flaccus $(8,92 \mathrm{sq})$, l'amour pur et légitime liant Médée au serpent va être brisé par l'arrivée de Jason, le tentateur, qui séduit Médée par une irrésistible passion. Nous retrouvons chez Avit le même triangle relationnel, avec une inversion des rôles entre le serpent et l'homme. Ce jeu allusif intertextuel est tout à fait dans l'esprit précieux que revendique Avit en tant que poète, lui qui, dans sa lettre $51^{45}$, se place en digne héritier de son oncle Sidoine Apollinaire, le plus éminent représentant de la poésie érudite de la Gaule de la fin du $5^{\text {ème }}$ siècle ${ }^{46}$.

Si, chez Ovide, le sens de l'expression est clair, Médée alternant un sentiment d'amour pour Jason et un sentiment de peur face aux épreuves qu'il doit affronter, en revanche, dans le texte d'Avit, ce sens est nettement moins obvie : la peur qu'éprouve Ève est bien évidemment celle des conséquences possibles de la transgression du commandement divin, mais de quel amour s'agit-il ? Est-ce l'amour conjugal par lequel Dieu l'a unie à son mari ( 1,185 : iusto constrictus amori maritus), et que n'a pas manqué de lui rappeler le serpent au commencement de son discours de séduction (2, 150: iure tuo subiectus amori), cet amour qui aurait dû lui interdire de prêter l'oreille au tentateur, comme l'exprime avec indignation le poète (v. 162-165); ou est-ce l'amour filial qu'elle doit au Père créateur, que souligne aussi le serpent en utilisant le nom pater lorsqu'il parle de Dieu à Ève ${ }^{47}$, et qui devrait la pousser à l'obéissance ? Ou est-ce, enfin, le désir coupable, la uoluptas de croquer une pomme rendue irrésistible par le serpent, et de devenir ainsi l'égale des créatures divines comme le lui promet ce dernier (v. 198-203) ? Le contexte dans lequel Avit insère cette expression amènerait plutôt à envisager ce dernier sens : en effet, il dit précédemment que des sentiments contraires envahissent l'esprit d'Ève (v. 221 : rapiunt contraria mentem) puis il précise qu'elle est tenaillée entre arrogance et obéissance à la loi divine (v. 222-223 : pulsat iactantia legem / interdumque etiam lex subuenit), cette loi que le serpent voulait briser par la uoluptas (v. 199 : nec captiua diu frenetur lege uoluptas).

Or, cette connotation négative de amor désignant un amour interdit se retrouve dans la poésie érotique, où le nom est opposé à pudor qui indique la retenue légitime que devraient observer les amoureuses malheureuses. Phèdre s'adresse ainsi à Hippolyte lorsqu'elle veut lui écrire : pudor est miscendus amori (ov. Epist. 4, 9); de même Byblis dit à son frère : coget amor....uel si pudor ora tenebit (ov. Met. 9, 515); et enfin, on ne saurait oublier cette opposition dans le chant 4 de l'Énéïde où Virgile conclut les paroles d'Anna convainquant Didon de céder à sa passion pour Ėnée par ces deux vers $(4,54-55)$ :

hic dictis impenso animum flammauit amore

spemque dedit dubiae menti soluitque pudorem

29 C'est d'ailleurs ce même pudor que Marius Victor oppose à timor lorsqu'il décrit les hésitations d'Ève par une variation, sans équivoque, sur l'expression ovidienne reprise par Avit ${ }^{48}$.

30 Le dernier protagoniste est donc le serpent. Je ne reviendrai pas sur la réutilisation chrétienne de ce reptile qui, dans la mythologie profane, est associé à un grand nombre de légendes, telle celle du jardin des Hespérides où il garde les pommes d'or ${ }^{49}$, mais je me contenterai de rappeler que le serpent participe aussi à des histoires d'amours tragiques, comme nous l'avons vu en ce qui concerne le dragon gardien de la toison d'or et cher à Médée. Dans le récit de la tentation que propose Avit, le serpent joue le premier rôle en tant que protagoniste principal du chant 2, ce qui est une innovation par rapport au texte biblique et aux épopées bibliques précédentes. 

caractérise le souffle de la brise agrémentant le paradis ${ }^{59}$. Ainsi, la voix caressante du serpent évoque l'arme absolue du séducteur, les blanditiae, au pouvoir irrésistible, que ce soit les caresses physiques, ou le charme de la voix ${ }^{60}$. Il suit donc à la lettre les recommandations d'Ovide (Ars 2, 159-160):

Blanditias molles auremque iuuantia uerba / adfer.

Une première remarque concerne le nom : draco est le seul nom utilisé pour désigner l'animal mythique, que ce soit celui qui garde le jardin des Hespérides (VERG.Aen. 4, 484) ou la Toison d'or (ov. Epist. 12, 101. 171 ; VAL. FLACC. 8, 60. 71. 92) ) $^{50}$ Avit quant à lui utilise draco lorsqu'il décrit l'animal dans un style très élevé, mais il connait aussi, par désir de variatio, serpens (selon Genèse 3, 6), coluber ou anguis ${ }^{51}$.

Avit a créé son personnage en reprenant un certain nombre de traits empruntés au séducteur de la poésie érotique. Le premier trait du serpent est sa « ruse mauvaise conseillère" (malesuada fraus au v. 136). Prudence utilise exactement la même expression au même endroit dans le vers dans ditt. 1, 2. Comme le remarque Deproost, si au début du récit de la tentation, l'adjectif malesuadus qualifie la ruse du serpent, à la fin de la scène, il s'applique au murmure par lequel Ève convainc Adam de manger la pomme, marquant ainsi l'achèvement de la tentation et illustrant le fait que de tentée, Ève devient à son tour tentatrice pour Adam ${ }^{52}$. Aux dires d'Ovide, le fraus est le reproche principal que l'on peut adresser aux séducteurs profitant de la credulitas féminine, à l'exemple du fallax Jason abandonnant Médée pour une autre femme ${ }^{53}$. Or, Avit insiste souvent sur ce dernier qualificatif lorsqu'il parle de l'ange déchu qui prend la forme du serpent ${ }^{54}$. Et si dans la poésie élégiaque - qui présente le point de vue de l'amant - la mention de la perfidie amoureuse est assez rare ${ }^{55}$, en revanche, c'est le reproche usuel qu'adressent à leur amant les amoureuses délaissées d'Ovide ${ }^{56}$. Ainsi, c'est sur l'opposition entre la fraus de l'amant/tentateur et la credulitas de la femme séduite que s'articule, comme dans la poésie érotique, la scène de la tentation.

Grâce à sa perfidie, le serpent trouve les moyens adéquats pour séduire Ève. Nous avons vu précédemment qu'il utilise d'abord les paroles pour atteindre le faible esprit de la femme. En effet, le premier artifice du diable qui a pris l'apparence d'un serpent est de faire de ses sifflements une sorte de chant destiné à charmer son interlocutrice, comme le faisait le dragon gardien de la toison d'or lorsqu'il parlait à Médée ${ }^{57}$.

Ainsi, pour que ses paroles soient très persuasives, sa voix se fait douce (leni uoce au v. 144) et caressante (blanditam uocem au v. 161), devenant même un murmure susurré à l'oreille complaisante (fallaci susurro au v. 204) d'une Ève presque conquise par les belles promesses (2, 204-205):

talia fallaci spondentem dona susurro

credula submisso miratur femina uultu.

Nous avons là la reprise de l'image type de l'entreprise de séduction masculine contre laquelle Properce met en garde Cynthie $(1,11,13-15)$ :

uacet alterius blandos audire susurros

molliter in tacito litore compositam

ut solet amota labi custode puella

perfida, communis nec meminisse deos $!^{58}$

Par ce murmure, la sensualité du séducteur est en phase avec la sensualité du lieu. En effet, comme dans la poésie lyrique profane, susurrus est la métaphore usuelle qui

\section{méthode de séduction du serpent est tout à f}

La méthode de séduction du serpent est tout à fait conforme à celle décrite dans la poésie érotique : après avoir charmé Ève par ses paroles, il va joindre le geste à la 
parole. En donnant ainsi un rôle très actif au serpent dans le processus de la tentation, Avit innove totalement par rapport au récit biblique. En effet, dans la Genèse, nous avons d'abord le dialogue serpent - Ève $(3,2-5)$, ensuite le serpent disparaît de manière aussi subite qu'il était apparu ${ }^{61}$, puisque l'action se focalise sur Ève qui voit le bel arbre, cueille le fruit, le mange et le donne à son mari (Genèse 3,6) :

Vidit igitur mulier quod bonum esset lignum ad uescendum et pulchrum oculis aspectuque

delectabile et tulit de fructu illius et comeditdeditque uiro suo qui comedit.

C'est donc elle qui est active dans toute cette scène. Or, dans le poème d'Avit, la situation est différente: outre l'amplification par laquelle le poète expose avec précision le décor dans lequel vont évoluer les protagonistes, (v. 136-144) et le long dialogue de persuasion (v. 145-203), Avit innove, d'une part, en caractérisant chacun des deux protagonistes ainsi que nous venons de le voir, et, d'autre part, en décrivant avec minutie le moment du geste fatal.

\section{Le fruit}

La première différence, et la plus connue, par rapport au texte biblique est la précision sur la nature du fruit, une pomme. Ce choix est dicté à la fois par des raisons de cohérence interne du texte, par l'imitatio ueterum et par un choix stylistique.

En effet, précédemment, nous avons vu que la scène se situe dans le cadre lyrique d'un verger où les premiers hommes cueillent des pommes rouges. Il semble dès lors logique que les hauteurs que gagne le serpent (v. 143: arboris erectae spiris reptantibus alto) soient celles d'un pommier, et que soit ainsi précisé la nature de l'arbre et du fruit de la Genèse.

41 Par ailleurs, il se trouve que la pomme joue un rôle important dans la mythologie gréco-romaine, en apportant l'immortalité comme l'indique la légende du jardin des Hespérides $^{62}$. Or, c'est bien l'immortalité, accompagnée de toutes les autres prérogatives divines, que promet le serpent à Ève (v. 200-203). De plus, la pomme est le fruit de l'amour, comme le prouvent les occurrences du nom malum dans la poésie érotique : c'est une pomme qui retarde la course d'Atalante ${ }^{63}$; ce sont des pommes que s'offrent les époux, dans les épithalames ${ }^{64}$.

Enfin, le nom latin de la pomme mālum (en fin des vers 139 et 210) est identique, à la quantité de la voyelle initiale près, à celui du mal, mălum (employé au v. 218 dans l'expression mali pondere ${ }^{65}$ ), ce qui permet au poète de qualifier la pomme de miserum munus au v. 213. En fait, la plus ancienne attestation du jeu de mots mālum/mălum se trouve dans la réponse adressée au poète Naevius par les Metelli, qu'il avait critiqués dans une épigramme à double sens : Malum dabunt Metelli Naeuio poetae ${ }^{66}$. Ce jeu topique est appliqué au fruit défendu de la Genèse dans PS. CYPRIENGenesis 77, où le serpent s'adresse ainsi à Ève :

dic mihi cur metuas felicia germina māli ?

numquid poma Deus non omnia nota sacrauit ?

43 Je crois que c'est grâce à la très grande diffusion du poème d'Avit qui a repris le jeu de mots du PS. CYPRIEN - dans le monde médiéval que le fruit défendu de la Genèse a été définitivement associé à une pomme ${ }^{67}$.

Cette ambiguïté du sens de malum, si elle est ici un jeu poétique volontairement recherché, est aussi signalée, ailleurs, par les exégètes chrétiens comme une confusion 
à éviter. En effet, dans le Cantique des cantiques, texte interprété comme un poème d'amour chrétien symbolisant les noces du Christ et de l'Église, un arbre appelé malum est mentionné à plusieurs reprises ${ }^{68}$. Quoi qu'il en soit de la nature exacte du fruit (il peut, en effet, s'agir d'une grenade, appelée en latin malum granatum, comme le comprend par exemple Apponius dans son Commentaire au Cantique des cantiques ${ }^{69}$ ),dans son exégèse du poème, Rufin met en garde contre l'équivoque liée aux deux sens de malum ${ }^{70}$.

En fait, si la nature exacte de l'arbre de la Genèse a suscité bien des interprétations - cep de vigne pour certains, figuier pour d'autres ${ }^{71}-$, dans les premières représentations de la scène, la nature stylisée de l'arbre ne permet pas l'identification du fruit. Ensuite, après le $\mathrm{VI}^{\mathrm{e}}$ siècle, étant souvent représenté comme un fruit rond, il devient une pomme, sous l'influence des représentations d'anciens mythes profanes comme celui d'Hercule cueillant des pommes dans le jardin des Hespérides.

\section{Le processus de la tentation}

Après avoir précisé la nature exacte du fruit défendu, Avit innove encore par rapport au récit biblique en décrivant longuement comment le serpent va aider Ève qui, bien que déjà vaincue par ses paroles, hésite encore à passer à l'acte. C'est pourquoi il décrit étape par étape avec minutie comment le serpent va captiver tous les sens de la femme afin de la faire succomber. Après avoir charmé son ouïe de son murmure trompeur (v. 204), c'est lui qui prend l'initiative de cueillir une pomme : la pesanteur du v. 210 avec son accumulation de spondées illustre bien la lenteur de l'approche et tandis que l'adjectif letali à la penthémimère du vers marque tout la solennité de l'acte mortel, le rejet du verbe detrahit au début du vers suivant rend la soudaineté du geste lui-même ${ }^{72}$. C'est alors que, pour charmer la vue de sa victime, le serpent lui présente un beau fruit (pulchrum thématisé par la césure du vers 211), puis, afin de solliciter son odorat, il enveloppe la pomme d'un suave parfum. A la différence du texte biblique, cette première étape fait du serpent l'acteur et le maitre de la scène et donne à la pomme un rôle central parce qu'elle est, à l'initiative du serpent, pourvue de tous les charmes nécessaires à la séduction érotique.

Après avoir ainsi focalisé sa description sur les préparatifs du serpent (v. 209-212), le poète se concentre ensuite sur la réaction d'Ève qui devient le sujet des verbes suivants (v. 213-216). Mais là encore, Ève reste passive, puisqu'elle hésite longuement, tantôt portant le fruit à sa bouche, tantôt retenant sa main. Pour que s'accomplisse l'acte, il faut que le serpent reprenne l'initiative en se penchant vers elle pour lui montrer avec insistance le fruit, dans une attitude qui rappelle celle du séducteur penché sur sa bienaimée $^{73}$. C'est encore lui qui l'incite ${ }^{74}$ à succomber, ce qu'Avit exprime par la prolepse ruina, nom par lequel Médée qualifiait déjà rétrospectivement son amour naissant pour Jason $^{75}$. Ce n'est qu'à ce moment-là, que définitivement vaincue, Ève prend elle-même la pomme (sumeret ipsa à la fin du vers 230).

Cette description littéraire n'est pas sans lien avec la représentation iconographique de la scène. En effet, d'après l'étude faite par Pascale Robin ${ }^{76}$, la scène, reproduisant les modèles mythologiques profanes, montre soit Adam et Ève de part et d'autre d'un arbre stylisé, à la nature non précisée, autour duquel est enroulé le serpent qui est relégué au second plan $^{77}$, soit, en suivant fidèlement le texte biblique, une Ève, le plus souvent nue et sans parure, cueillant la pomme. P. Robin relève en particulier le parallélisme 
frappant entre la scène de la tentation et certaines scènes représentant Jason et Médée de part et d'autre du serpent enroulé autour d'un arbre, ce qui l'amène à conclure que "la disposition des deux héros, la présence de l'arbre et du reptile présentent des analogies trop frappantes avec la scène «imaginée » et représentée par les chrétiens pour qu'on ne doive pas supposer de rapport entre les deux compositions. $»^{78}$

Il existe des fresques plus singulières, en correspondance avec la vision de la scène qui se dégage du texte d'Avit. Sur un sarcophage du début du IV siècle, on voit une Ève parée de bijoux, avec une coiffure compliquée, accentuant ainsi les traits de séduction de la femme qui est représentée en train de donner le fruit à son mari ${ }^{79}$. Un tel tableau coïncide avec l'atmosphère dégagée par le texte d'Avit qui avait insisté sur la beauté remarquable d'Ève "prête pour le mariage " dans le récit de sa création ${ }^{80}$ et elle correspond à l'analyse que je propose de cette scène, inspirée de la poésie érotique. Encore plus près de notre texte, sur un sarcophage gaulois provenant de la ville d'Auch, datant de la fin du vie siècle, «le serpent, tête pointée vers Ève, tient un fruit dans sa gueule, qu'il semble lui offrir : le tentateur paraît avoir détaché lui-même le fruit de l'arbre, ce qui n'est pas sans minimiser quelque peu la culpabilité d'Ève, qui se contente d'accepter ce que le reptile lui offre, sans avoir fait le geste décisif de le cueillir ${ }^{81}$. On ne peut que souligner la ressemblance frappante entre les représentations littéraire et iconographique de la scène. Toutefois, je crois qu'une telle convergence n'est pas fortuite, car j'ai relevé par ailleurs, à propos des chants 4 et 5 , «l'esthétique picturale » du poème d'Avit qui propose de petits médaillons de différentes scènes bibliques. Il reste à faire une étude détaillée des rapports précis entre ces descriptions littéraires et les représentations iconographiques correspondantes ${ }^{82}$.

\section{Le sens du récit d'Avit}

En donnant ainsi le rôle principal au serpent, Avit réduit considérablement celui d'Ève qui, victime faible et passive, n'est donc qu'un simple intermédiaire dans cette tentation. Cette analyse correspond à l'exégèse qu'ont donnée de la scène les commentateurs chrétiens, en particulier Ambroise et Augustin.

51 À la fin du livre $11 \mathrm{du}$ De genesi ad litteram, Augustin cherche à comprendre le processus du premier péchés3. Puisqu'Adam, en tant qu'être spirituel, ne peut être tenté directement par le serpent, Ève joue le rôle d'intermédiaire parce qu'elle possède une intelligence plus faible et vit « selon l'inclination de la chair et non selon le mouvement de l'esprit $"^{84}$. Dans cette perspective, la faute d'Adam est "une condescendance injustifiée à l'égard de son épouse, l'effet d'une bienveillance amoureuse ${ }^{85}$ et la faute originelle est donc un «désordre dans l'amour conjugal». Une telle interprétation distingue donc une psychologie différente entre les deux êtres humains : la sensibilité de la femme est la médiation nécessaire à l'introduction du péché dans Adam. Déjà Ambroise $^{86}$, reprenant l'exégèse de Philon, avait distingué la compréhension rationnelle, mens ou " noûs » d'Adam de la compréhension sensible, sensum ou « aisthèsis » d'Ève et relevé que la prévarication n'avait été possible que per uoluptatem et sensum.

Donc, en construisant son récit de la tentation comme une scène de séduction amoureuse, Avit développe de manière poétique l'exégèse augustinienne du péché originel. 

décrivant Ève séduite sous les traits d'une femme en proie
l'égarement de l'amoureuse séduite puis délaissée $(2,238-39)^{89}$ : audacia primum flabat femineos animosa in corda furores.

\section{Conclusion}

En empruntant à la poésie érotique profane ses principaux traits spécifiques, Avit, bien loin du récit biblique, construit une véritable psychologie de ses deux protagonistes principaux : par là, il introduit, de manière quelque peu inattendue dans une épopée biblique, la composante érotique, souvent présente dans l'épopée classique depuis Virgile. En fin connaisseur de la littérature antique, il le fait habilement en utilisant L'opposition entre le processus de la tentation et la communion se poursuit dans les
conséquences de l'ingestion. Si, par l'eucharistie, le fidèle rencontre Dieu et le bien, en écrivant Ève séduite sous les traits d'une femmen proie à un furor qui évoque 
l'allusion, et, en évêque soucieux de l'orthodoxie du dogme, sur le fond, il suit l'exégèse augustinienne du péché originel.

En ce qui concerne les modèles classiques utilisés par le poète, il apparait, aussi bien d'après les parallèles textuels que d'après les rapprochements extérieurs que l'on peut faire avec les représentations iconographiques, que la tentation est construite en référence avec l'une des plus célèbres scènes de séduction mythologiques, la rencontre entre Médée et Jason près de la Toison gardée par le serpent. En effet, je crois que le poète crée sa scène non pas en référence à un auteur particulier, mais en pensant à la rencontre entre Médée et Jason connue par la tradition littéraire, principalement dans la lettre 12, dans le début du chant 7 des Métamorphoses d'Ovide et le chant 8 des Argonautiques de Valerius Flaccus. Même s'il y a un lien évident entre la Didon virgilienne et l'Ève d'Avit, la structure de l'ensemble de la scène et le rôle essentiel du serpent/tentateur conduisent plutôt à voir en Ève une réécriture originale de la Médée amoureuse des poètes classiques.

Dans ce dialogue avec ses modèles, le poète transpose consciemment le rôle des protagonistes. Si Ève, comme Médée, est la femme séduite qui abandonne ces liens familiaux pour obéir à son séducteur, ce n'est pas l'homme Adam qui endosse le rôle de Jason, le séducteur, mais le serpent qui devient le protagoniste principal alors qu'Adam reprend le rôle très effacé du dragon, gardien de la Toison. Cette variatio de la triangulation classique est soulignée dès le tout début de la rencontre entre le serpent et Ève. En effet, après le séduisant discours du serpent, avant de donner la parole à Ève le poète clame, dans une courte diatribe indignée, son étonnement et sa stupéfaction, d'une part d'entendre un animal parler, et d'autre part, de voir Ève répondre à ces paroles inattendues $(2,162-165)$ :

Quis stupor, o mulier, mentem caligine clausit ?

Cum serpente loqui, uerbum committere bruto

non pudet, ut uestram praesumat belua linguam?

Et monstrum pateris, responsumque insuper addis ?90

60 N'est-ce pas là un indice que donne le poète afin que le lecteur comprenne le jeu de l'allusion au modèle classique, si habilement transposée?

\section{BIBLIOGRAPHIE}

P. AGAËSSE et A. SOLIGNAC, La Genèse au sens littéral en douze livres, t. 2 (Bibliothèque augustinienne $\left.\mathrm{n}^{\circ} 49\right)$, Paris 1972

A. ARWEILER, Die Imitation antiker und spätantiker Literatur in der Dichtung De spiritalis historiae gestis des Alcimus Avitus, Berlin/NewYork, 1999

H. CROUZEL, Origène et la « connaissance mystique », Paris-Bruges 1961

W. DEONNA, Evodia, croyances antiques et modernes : l'odeur suave des dieux et des élus, Torino 2003 
P. A. DEPROOST,« La mise en scène d'un drame intérieur dans le poème Sur le péché originel d'Avit de Vienne », Traditio 51 (1996), p. 43-72

D. FASCIANO, « La pomme dans la mythologie gréco-romaine » dans J. B. CARON et alii, Mélanges Lebel, St-Jean Chrysostôme /Québec 1980, p. 45-55

J. FONTAINE, Naissance de la poésie dans l'occident chrétien, Paris 1981

B. FRAIGNEAU-JULIEN, Les sens spirituels et la vision de Dieu selon Syméon le nouveau théologien, Paris 1985

N. HECQUET-NOTI, Avit de Vienne, Histoire spirituelle, t. 1, SC 444, Paris 1999

S. LILJA, The Roman Elegists' Attitude to Women, Helsinki 1965

A. R. LITTLEWOOD, « The Symbolism of the Apple in Greek and Roman Literatur », HSCP 72 (1967), p. $147-181$

C. MOUSSY et C.CAMUS, Dracontius œuvres, t. 1, CUF, Paris 1985

M. ROBERTS, The Jeweled Style : Poetry and Poetics in Late Antiquity, Ithaca-London 1989

P. ROBIN, « Représentation iconographique de la faute d'Adam et Ève dans le premier art

chrétien » dans Romanité et cité chrétiennes (Mélanges Y. Duval), Paris 2000, p. 19-30

D. SHANZER et I. WOOD, Avitus of Vienne, Letters and Selected Prose, Liverpool 2002

B. DE VREGILLE et L. NEYRAND, Apponius, Commentaire sur le Cantique des cantiques, t. 3 (SC 430), Paris 1998.

\section{NOTES}

1. Dans la Genèse, ce récit occupe les versets 1-6 du chapitre $3:$ Sed et serpens erat callidior cunctis animantibus terrae quae fecerat Dominus Deus qui dixit ad mulierem : «cur praecepit uobis Deus ut non comederetis de omni ligno paradisi ? " 2. Cui respondit mulier: «De fructu lignorum quae sunt in paradiso uescemur; 3. de fructu uero ligni quod est in medio paradisi praecepit nobis Deus ne comederemus et ne tangeremus illud ne forte moriamur. " 4. Dixit autem serpens ad mulierem : «nequaquam morte moriemini. 5. Scit enim Deus quod in quocumque die comederitis ex eo aperientur oculi uestri et eritis sicut dii scientes bonum et malum. " 6. Vidit igitur mulier quod bonum esset lignum ad uescendum et pulchrum oculis aspectuque delectabile et tulit de fructu illius et comedit deditque uiro suo qui comedit. L'ensemble du récit d'Avit correspondant à ces versets occupe les vers 118 à 260 du chant 2.

2. N. HECQUET-NOTI, Avit de Vienne, Histoire spirituelle, t. 1, SC 444, Paris 1999, p. 40-51 (Sur les liens structuraux entre les 3 chants) et p. 177-181 (plan commenté du passage).

3. P. A. DEPROOST, «La mise en scène d'un drame intérieur dans le poème Sur le péché originel d'Avit de Vienne », Traditio 51 (1996), p. 43-72.

4. Je ne parle pas ici de la simple paraphrase de In Genesim ad Leonem papam d'un certain Hilaire (édité par PEIPER CSEL 23 p. 231-239) dont deux vers (v. 160-162) font allusion à l'ensemble de l'épisode sans mentionner Ève : «Après que le premier homme n'eut pas craint de se nourrir du bois défendu / et que, prisonnier de ses ruses, il s'offrit au serpent, / il se montre condamné... » (postquam primus homo uetito se pascere ligno / non timuit captusque dolis se praebuit angui, / stat reus... ) .

5. Alors que les autres épopées bibliques donnent brièvement la parole au serpent, Avit au contraire construit une très longue scène de dialogue entre Ève et le serpent $(2,136-203)$ : voir HECQUET-NOTI, Histoire spirituelle t.1 p. 177-181 et DEPROOST « La mise en scène ... ». 
6. Sur cette épopée, voir J. FONTAINE, Naissance de la poésie dans l'occident chrétien, Paris 1981, p. 246-247.

7. PS. CYPR.Gen. 81-84 : «Celle-ci refuse et craint de toucher aux rameaux défendus. Mais pourtant son cœur est vaincu par ses faibles sens. Aussitôt qu'elle eut mordu les doux fruits de ses dents de neige, le ciel serein, souillé d'aucun nuage, étincela ».

8. Sur Marius Victor, voir FONTAINE, Naissance de la poésie chrétienne p. 241-242; sur Dracontius, voir le même ouvrage p. 253-256 et l'introduction de c. Moussy et c. CAMUS, Dracontius œuvres, t. 1, CUF, Paris 1985, p. 7-91.

9. «En effet, après qu'Ève, dans sa crédulité, eut rompu, par une morsure sacrilège, les prescriptions édictées, instruite désormais du mal qu'elle a expérimenté, elle cherche une consolation à sa faute ".

10. Laud. dei 1, 469b-472: "c'est l'oreille de l'épouse qu'il assaille d'une voix douce. Traîtreusement, avec des paroles perfides, il s'adresse au cœur faible, hélas ! de la jeune fille : elle porte à sa bouche un aliment où ne réside que la mort cruelle »: traduction de c. CAMUS, Dracontius, œuvres t. 1, CUF, Paris 1985, p. 175.

11. «De la promesse de tels dons, exprimée en un murmure trompeur, la femme crédule, les yeux baissés, s'émerveille. Et dès lors, elle se met à hésiter, à laisser son coeur s'émouvoir et à rapprocher toujours plus de la mort son esprit hésitant. Quand il se rendit compte qu'elle était vaincue par le malheur qui la guettait, lui rappelant une fois encore le nom et la citadelle des êtres divins, le serpent cueille, parmi toutes celles de l'arbre porteur de trépas, une seule pomme qu'il agrémente d'un parfum suave. Il la rend belle à voir et lui offre sur ces entrefaites, alors qu'elle hésite; et cette femme qui se fie à lui pour son malheur, faiblit face à ce funeste cadeau, et, prenant de ses mains le fruit fatal, elle le retourne en tous sens. A plus d'une reprise, elle le porte d'elle-même à son nez et à ses lèvres grandes ouvertes et, ignorante, joue avec sa mort future. O combien de fois, l'ayant approché de sa bouche, pleine de remords, elle écarta le fruit ; sa main, chancelant sous le poids d'un forfait téméraire, se retira et, dans un tremblement, fuit devant l'accomplissement de son crime! Elle désire pourtant être semblable aux êtres divins, et le poison malfaisant de l'ambition s'insinue en elle. Des partis contraires déchirent son esprit: d'un côté, l'amour, de l'autre, la crainte. L'arrogance chasse le commandement divin et parfois le commandement vient encore à son secours. Le flot hésitant de son cœur partagé par de durs combats bouillonne. Et pourtant, le serpent tentateur ne renonce pas à l'abuser et, tandis qu'elle hésite, il lui montre avec insistance cette nourriture, se plaint de ses atermoiements et la seconde vers la déchéance d'une chute abrupte. Quand fut arrêtée en son esprit enfin vaincu la décision la pire, celle de courir le risque d'avoir faim à jamais en absorbant une nourriture criminelle et de rassasier le serpent du repas qu'elle prendrait elle-même, elle donna son assentiment à ce piège, mordit et dévora le fruit ».

12. Voir le titre de son article cité supra note 3.

13. Tous les poètes chrétiens décrivent ainsi le paradis sur le modèle du locus amoenus de la poésie profane (p. ex. PRUDENCE, Cath. 3, 21sqq ; 5, 113sqq) : voir HECQUET-NOTI, Histoire spirituelle, t. 1. p. 121-124.

14. V. 17-19: «voici encore de petits fromages que sèche une claie de jonc, voici les prunes blondes d'un jour d'automne, des châtaignes et des pommes doucement rougissantes »Cf. ecl. 1, 80-81 : sunt nobis mitia poma / castaneae molles. On retrouve aussi les rubentia mala dans un poème lyrique attribué à Pétrone (fgt 46,3: dant rami cerasos, dant mala rubentia siluae).

15. PROP. 1, 20, 35-40 : «Au dessus, venus d'eux-mêmes, sans aucun soin, des fruits couverts de rosée pendaient aux arbres solitaires; tout autour, dans une prairie arrosée, des lis, dont la blancheur se mêlait à la pourpre des pavots. Et l'enfant, d'un ongle tendre, se met à la cueillette : la fleur l'a rendu oublieux et négligent » (quam supra nulli pendebant debita curae / roscida desertis poma sub arboribus, / et circum irriguo surgebant lilia prato / candida purpureis mixta papaueribus. / 
quae modo decerpens tenero pueriliter ungui / proposito florem praetulit officio.) Traduction de D. Paganelli, CUF 1970.

16. 1, 231-232 : Nam quidquid nobis toto nunc nascitur anno, / menstrua maturo dant illic tempora fructu ( en effet, tout ce qui, maintenant, naît pour nous pendant le long d'une année, là-bas, chaque mois l'apporte à maturation. ») ; 1, 236-237 : sic cum desit hiems nec torrida ferueat aestas, / fructibus autumnus, uer floribus occupat annum (« Ainsi, puisque l'hiver n'existe pas et que les ardeurs de l'été ne sont pas brûlantes, de ses fruits l'automne comble l'année, le printemps de ses fleurs. ») 17. 1, 250:<flos> qui sparsus terris suaues dispensat odores ; 2, $211:<$ malum>detrahit et suaui pulchrum perfundit odore.

18. Par exemple CATUlle 13, 8-12 ; tibulle 3, 4, 28sqq ; 3, 8, 15sq ; PROPERCE 3, 10, 11sq et 3, 17, 29sqq : la femme amoureuse reçoit le parfum comme arme de séduction.

19. VERG., ecl. 2, 51-55 : «Moi-même je cueillerai les blanches pommes au tendre duvet, et les châtaignes, qu'aimait mon Amaryllis : j'y joindrai la prune vermeille (...). Et vous aussi, lauriers, myrtes si bien assortis, je vous cueillerai, puisqu'ainsi rassemblés vous confondez vos suaves odeurs ». Cette même expression, à une place différente dans le vers, se rencontrait déjà dans CATULLE 64, 87, autre poème exprimant le sentiment amoureux.

20. V. 140-144 : tum ueritus serpens, firma ne mente uirili / non queat iniecto subuertere corda ueneno, / arboris erectae spiris reptantibus alto / porrigitur tractumque suum sublimibus aequans / auditum facilem leni sic uoce momordit. Le verbe subuertere, très peu usité dans la poésie classique, exprime, chez les historiens, le renversement complet de la situation de quelqu'un : cf. oLD. S.v qui renvoie p. ex. à TAC. Hist., 4,42.

21. V. 235-237 : « Ignorant le fait (scil. La séduction d'Ève), Adam, joyeux, s'en revenait d'un côté opposé à travers la vaste campagne herbeuse, en cherchant l'étreinte de son épouse et ses chastes baisers. " (Ignarus facti diuersa parte reuertens / Adam diffusi laetus per gramina campi / coniugis amplexus atque oscula casta petebat.)

22. Présence du mari dans l'élégie : p. ex. dans TIBULle 1,$2 ; 1,6$; OVIDE, am. 1, 4;3, 4 .

23. DEPROOST, « la mise en scène... » p. 67.

24. Voir P. AGAËSSE et A. SOLIGNAC, La Genèse au sens littéral en douze livres, t. 2 (Bibliothèque augustinienne ${ }^{\circ} 49$ ), Paris 1972, Note complémentaire p. 555-559 «le processus du premier péché ».

25. AUGUSTIN, conf. 2, 3, 8 : calcabat me inimicus inuisibilis et seducebat me, quia ego seductilis eram.

26. ovidE, Met. 3, 379 (en parlant de Narcisse dans la légende d'Écho) : forte puer comitum seductusab agmine fido. Chez Sénèque, il qualifie le sage qui se retire à l'écart du monde (Epist. 55,4) et DRACONTIUS l'utilise pour qualifier Ève (Laud. Dei 1, 477).

27. La même expression est reprise dans Epist. 6, 21 (parole adressée à Jason par Hypsiphyle). Ce topisme se trouve aussi dans l'élégie : p. ex. PROPERCE, 2, 21, 6 ; TIBULLE 3, 10, 18. Il est vrai que tout amant, homme ou femme, tombe dans cette naïveté amoureuse (voir ovIDE, Am. 3, 14, 30), mais c'est avant tout un reproche formulé par les amoureuses abandonnées.

28. De la même manière, Phèdre oppose sa crédulité à l'amour trompeur d'Hippolyte: o spes amantum credula, o fallax amor! (SEN. Phaedr. 634).

29. MAR. VICTOR. Aleth. 1, 411. Dracontius applique le même qualificatif aux cœurs des deux êtres humains qu'il unit dans l'évocation de la tentation : credula corda reatum / incurrant (Laud. Dei 1, 466).

30. V. 205 : credula...femina, puis plus loin encore, mulier male credula (v. 213).

31. 3, 103 : credulus ipse fui, sed credere tu docuisti / conubium donans.

32. V. 206-207: Et iam iamque magis cunctari ac flectere sensum / incipit et dubiam leto plus addere mentem.

33. 7, 171-173a: «Je ne t'ai pas fait défaut, quand tu as entrepris de fléchir les sens de la jeune fille et de toucher son âme par un souci inconnu ». 
34. Argonautiques, 7, $176-178$.

35. 2, 221b-224 : « Des sentiments contraires déchirent son esprit : d'un côté l'amour, de l'autre la crainte. L'arrogance chasse la loi divine, et, parfois, cette loi vient encore à son secours. Le flot hésitant de son cœur partagé par de durs combats bouillonne. »

36. Voir les nombreux parallèles cités par DEPROOST p. 64-65 : v. 206-207 / Aen. 4, 22-23. 55 ; v. 220-221 /Aen. 4, 66-67 et 285-287 ; v. 222-223 /Aen. 4, 27 et 281-282; v. 228 / Aen. 4, 287.

37. Le groupe était déjà présent dans Met. 9, 473 avant le discours de Byblis.

38. Avit, 2, 223 et ov. Met. 9, 465.

39. Voir p. ex. TIB. 1, 3,19; 1,9, $41 ; 2,3,17$; PROP.1, 10, 4 ; ov. Met. 3, 375 (E்cho hésite à s'adresser à Narcisse).

40. ov. Met. 10,661 .

41. À la fin du v. 210 mālum, comme chez Ovide, signifie pomme. Le groupe pondus măli se lit également dans SEN. Herc. 0. 231.

42. Pour ce qui est de la variation entre hinc... hinc chez Ovide et hinc...inde chez Avit, il ressort de la consultation du CDRom Poetria Nova que les poètes augustéens utilisent exclusivement hinc.. hinc et ne connaissent absolument pas hinc...inde, qu'affectionnent les poètes chrétiens (en particulier Dracontius et Vénance Fortunat).

43. «C'est en vain, Médée, que tu résistes ; Je ne sais quel dieu s'oppose à toi ; » se dit-elle, «il serait surprenant que ce ne soit pas (à moins que cela n'y ressemble beaucoup) ce qu'on appelle aimer. En effet, pourquoi les ordres de mon père me semblent-ils trop durs ?Ils sont vraiment trop durs. Pourquoi, celui que je viens enfin de voir, je crains qu'il ne périsse ? Quelle est la cause d'une si grande crainte?»

44. La linguae gratia ficta de Jason (Epist. 12,14) rappelle 2, 161 : haec male blanditam finxerunt sibila uocem (à propos du serpent) et la ruse utilisée par Jason (v. 52 ultimus est aliqua decipere arte labor) pour tromper le serpent gardien de la toison est celle qu'utilise le serpent pour séduire Ève.

45. Avit, epist. 51, p. 80, 35-81, 3 (Peiper in MGH AA, VI,2) : voir désormais la traduction anglaise de D. SHANZER et I. WOOD, Avitus of Vienne, Letters and Selected Prose, Liverpool 2002, p. 342-348 et la mise au point concernant la fin de la lettre dans N. HECQUET-NOTI, « Faut-il lire senem Arcadium dans la lettre 51 d'Avit de Vienne », MH 62 (2005), p.148-161. Sur la réception des auteurs classiques dans Avit, voir A. ARWEILER, Die Imitation antiker und spätantiker Literatur in der Dichtung De spiritalis historiae gestis des Alcimus Avitus, Berlin/New York, 1999.

46. Voir l'ouvrage classique de A. LOYEN, Sidoine Apollinaire et l'esprit précieux en Gaule aux derniers jours de l'Empire, Paris 1943.

47. 2, 187: sed pater inuisus sortem non contulit aequam ; 2, 197: ...quaecumque pater secreta reponit.

48. MARIUS VICTOR, Aleth. 1, 423 : hinc timor, inde pudor.

49. VERG. Aen. 4, 484sqq fait allusion à cette légende.

50. Dans Ov. Epist. 12, 196, Médée utilise l'expression serpens uicta de manière péjorative.

51. Draco en 2, 123. 183 ; serpens p. ex. en 2, 140. 181. 225 ; anguis en 2, 137. 233 ; coluber en 2,130. 169 (voir J. RAMMINGER, Concordantiae in Alcimi Ecdicii Avitii carmina, Hildesheim 1990).

52. DEPROOST «La mise en scène.. ", p. 57sq. PAUL. NOL. carm. 24, 553 parle de malesuada uerbis fraudis arte pour la même action.

53. C'est exactement ce que dit Médée à Jason dans ov. Epist. 12, 120 : tu fraudis poenas, credulitatis ego ; voir aussi Ars 3, 31- 33 .

54. 2, 61 ora...fallentia; 2, 67 auri fallentis amore; 2, 113 fallendi causa ; 2, 204 fallaci susurro ; 2,225 nec...desistit fallere serpens. On peut aussi relever les autres qualificatifs appartenant au même champ sémantique : simulata figura $(2,72)$ altior astu $(2,118)$; callidus $(2,183.233)$.

55. Tibullene l'utilise pas et Properce l'applique aux femmes (p. ex. en 2, 9, 31) en référence à Circé $(3,12,27)$ 
56. On rencontre une allusion à la fraus presque dans toutes leurs lettres (p. ex. 2, 78; 6, 163; 10, $76 ; 12,91 ; 20,24 ; 21,129)$.

57. 2, 134-135: "Maintenant, il feint d'être caressant, sa gorge s'amuse à une sorte de chant continuel et sa gueule agite en tous sens sa langue à trois pointes » (nunc simulat blandum, crebro ceu carmine fauces / ludunt et trifidam dispergunt guttura linguam). Voir VALERIUS FLACCUS, 8, 63 : ...et blanda poscit me pabula lingua.

58. «Garde-toi de prêter l'oreille à des murmures caressants, dans une pose nonchalante et coquette sur le rivage silencieux : ainsi loin de toute surveillance, tombe la femme qui se parjure, oubliant les serments et les dieux » (Traduction de D. Paganelli CUF, p. 19).

59. Avit, 1, 248 : flatibus exiguis lenique impulsa susurro / diues silua rappelle le décor bucolique de VERG. Ecl. 1, 55 : saepe leui somnum suadebit inire susurro, décor auquel Avit se réfère par l'évocation des hommes cueillant des pommes (voir supra). L'expression lenis susurrus se trouve dans HoR. carm. $1,9,18$; voir aussi Culex $105 ; 121 ; 156$ ou TIBERIANUS, carm. $1,16$.

60. P. ex. PROP. 1, 9, 29-30: quisquis es, adsiduas a fuge blanditias! / illis et silices et possint cedere quercus (il s'agit de caresses physiques) , тіВ. 1, 2, 93 : Et sibi blanditias tremula componere uoce ou ov. Am. 2, 1, 21 : blanditias elegosque leuis, mea tela, resumpsi (il s'agit de la voix). Voir s. LILJA, The Roman Elegists' Attitude to Women, Helsinki 1965, p. 204sq.

61. Les seules informations concernant le serpent sont celles de $3,1:$ sed et serpens erat callidior cunctis animantibus terrae quae fecerat Dominus Deus.

62. Cf. D. FASCIANO, « la pomme dans la mythologie gréco-romaine » dans J. B. CARON et alii, Mélanges Lebel, St- Jean Chrysostôme /Québec 1980, p. 45-55 et A. R. LITTLEWood, "The Symbolism of the Apple in Greek and Roman Literatur ", HSCP 72 (1967), p. 147-181.

63. ov. Met. 10, 677 (voir supra) et VERG. Ecl. 6, 61.

64. P. ex. CATVll. 65, 19 ; Claudien, Carm. min. 25, 15 (épithalame de Palladius et Celerina). APuléE, Met. 10,32 la mentionne dans une parodie de cortège d'épithalame (voir ThlL 8, 209, 57sq).

65. Cf. supra la discussion à propos de cette expression reprise d'Ovide.

66. Voir A. ERNOUT, Recueil de textes latins archaïques, Paris 1938, p. 140, 29n.

67. Sur la large diffusion du poème dans le monde médiéval et sa présence constante dans les canons des poètes latins chrétiens lus au Moyen-Âge, voir HECQUET-NOTI, Histoire spirituelle, t. 1, p. $86-92$.

68. 2,3 : sicut maluminter ligna siluarum sic dilectus meus....fructus eius dulcis gutturi meo, 8, 5 : sub arbore malo (d'autres exégètes lisent mali).

69. APP. 12, 3, ou encore GRÉGOIRE D'ELVIRE in cant. 3, 21-22 : voir B. DE VREGILLE et L. NEYRAND, Apponius, Commentaire sur le Cantique des cantiques, t. 3 (SC 430), Paris 1998, note 1, p. 170-171.

70. RUFIN Orig. In cant. 3, 5, 2 (SC 376 p. 524).

71. Dans la tradition juive, c'est un cep; dans la Septante et pour les premiers exégètes chrétiens grecs, un figuier. Voir en dernier lieu l'article de P. ROBIN, « Représentation iconographique de la faute d'Adam et Ève dans le premier art chrétien » dans Romanité et cité chrétiennes (Mélanges Y. Duval), Paris 2000, p. 19-30.

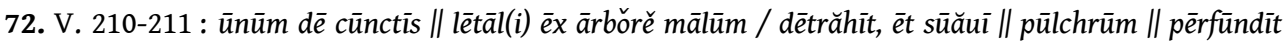
ǒdōrě.

73. PROP.1, 3,19-26

74. Le poète emploie le nom rare et tardif incentor, qui, d'après ThlL VII, 1, 874, 14sq, est utilisé, la plupart du temps, in malam partem, en particulier pour désigner le diable chez les chrétiens.

75. V. 225. $227:$ nec ...incentor desistit fallere serpens/....et iuuat in lapsum pendentis prona ruinae. Pour ce dernier mot, voir ovidE, Epist. 12, 34.

76. Voir l'article cité note 71.

77. C'est le cas de la première représentation connue (avant 256) sur le baptistère de Doura Ouropos, puis dans la catacombe de Domitilla (vers 315). 
78. Sur l'iconographie profane de la scène Médée, Jason et le serpent, voir V. GAGGADIS-ROBIN, Jason et Médée sur les sarcophages d'époque impériale, Ėcole française de Rome 1994, p. 79-88.

79. P. Robin (p. 23) relève l'intention moralisatrice de telles représentations, où Adam, conscient de sa désobéissance, mais incapable de résister à sa tentatrice, est «représenté tête baissée, épaules courbées » (en particulier sur le sarcophage de Junius Bassus datant de 359, ou sur un fond de verre de Cracovie).

80. Voir 1,156 : erigitur pulchro genialis forma decore.

81. Je cite P. Robin, p. 29.

82. Voir les remarques dans HECQUET-NOTI, Histoire spirituelle, t. 2 , Paris 2005, p. 19-20 et 118-119. Une esquisse générale de la question se trouve dans M. ROBERTS, The Jeweled Style: Poetry and poetics in late Antiquity, Ithaca-London 1989, p. 66-121 (chapitre intitulé « Poetry and the Visual Arts »).

83. Voir P. AGAËSSE et A. SOLIGNAC, Note complémentaire «le processus du premier péché » citée supra note 24 .

84. Je cite Agaësse et Solignac.

85. Je cite Agaësse et Solignac.

86. AMBR. De parad. 2,11

87. C. Cels. 1, 48. Voir B. FRAIGNEAU-JULIEN, Les sens spirituels et la vision de Dieu selon Syméon le nouveau théologien, Paris 1985, p. 29-42 et H. CROUZEL, Origène et la «connaissance mystique ", Paris-Bruges 1961.

88. Cf. W. DEONNA, Evodia, croyances antiques et modernes: l'odeur suave des dieux et des élus, Torino 2003 : l'encens est le parfum privilégié de la communication divine, car, déjà dans la religion profane, sa subtilité est en accord avec la nature de la divinité.

89. P. ex. Ariane (CATUlLE, 64, 54), Didon (VERG. Aen. 4,501) ou Médée (SEN. Med. 392).

90. "Quel engourdissement, femme, a enfermé ton esprit dans les ténèbres? Tu n'as pas honte de parler avec un serpent, d'échanger des paroles avec un animal, à supposer qu'une bête emprunte votre langue ? Tu supportes ce prodige et, de plus, tu réponds?».

\section{RÉSUMÉS}

Quand il décrit la tentation d'Ève par le serpent dans son épopée biblique, L'histoire spirituelle, Avit amplifie considérablement le récit de la Genèse en composant une scène très détaillée du geste fatal à Ève. Un examen attentif de la structure du passage montre que le poète réécrit une scène typique de la poésie érotique classique en se fondant sur les modèles élégiaques, en particulier sur les évocations de la rencontre entre Médée et Jason existant dans la poésie d'Ovide ou de Valérius Flaccus. Toutefois, même en pratiquant ce jeu d'intertextualité, le poète se conforme à l'exégèse chrétienne qui apparaît en particulier chez Ambroise de Milan. Enfin, cette description visuellement très détaillée trouve une correspondance dans l'iconographie chrétienne de la scène, elle-même fortement inspirée du mythe profane de Médée et Jason.

\section{INDEX}

Mots-clés : épopée biblique, intertextualité, poésie érotique romaine, tentation d'Ève, Médée et Jason 
AUTEUR

NICOLE HECQUET-NOTI

Université de Genève

Nicole.Hecquet@lettres.unige.ch 